\title{
Máxima fase estável de lactato sanguíneo e o limite superior do domínio pesado em ciclistas treinados
}

\author{
Maximal lactate steady state and the upper boundary of heavy- \\ intensity domain in trained cyclists
}

\author{
Luis Fabiano Barbosa 1 \\ Mariana Rosada de Souza 1 \\ Jailton Gregório Pelarigo 1 \\ Renato Aparecido Corrêa Caritá 1 \\ Fabrizio Caputo 1 \\ Benedito Sérgio Denadai 1 \\ Camila Coelho Greco 1
}

1 Universidade Estadual Paulista, Laboratório de Avaliação da Performance Humana, Rio Claro, SP. Brasil.

Recebido em 23/07/08 Revisado em 27/10/08 Aprovado em 13/02/09
Resumo - O objetivo deste estudo foi verificar se a máxima fase estável de lactato (MLSS) delimita o limite superior do domínio pesado em ciclistas bem treinados. Quatorze ciclistas do sexo masculino $(25,5 \pm 4,4$ anos, $69.5 \pm 7,8 \mathrm{~kg}, 175,8 \pm 7,5 \mathrm{~cm})$ realizaram, em diferentes dias, os seguintes testes: exercício incremental até a exaustão para a determinação do pico de consumo de oxigênio $\left(\mathrm{VO}_{2}\right.$ pico) e; 2 a 4 testes de carga constante com duração de até 30 minutos para a determinação da MLSS. O valor do $\mathrm{VO}_{2}$ obtido no 30 min de exercício foi estatisticamente maior que o valor obtido no 3 o min de exercício a 100\%MLSS (3379,3 $\pm 250,1$ vs. $3496,7 \pm 280,2 \mathrm{ml} / \mathrm{min}, \mathrm{p}<0,05)$ e a $105 \% \operatorname{MLSS}(3439,5 \pm 289,3$ vs. $3545,5 \pm$ $303,2 \mathrm{ml} / \mathrm{min}, \mathrm{p}<0,05)$. O VO obtido no 30 - min no exercício realizado tanto a $100 \% \mathrm{MLSS}$ quanto a 105\%MLSS foi significantemente menor do que o $\mathrm{VO}_{2}$ pico $(3978,6 \pm 296,2 \mathrm{ml} /$ min, $\mathrm{p}>0,05)$. Com base nestes resultados, pode-se concluir que durante o exercício pesado (MLSS) $\mathrm{O} \mathrm{VO}_{2}$ não apresenta estabilidade, tomando como referência os valores obtidos por volta do 3ำ minuto de exercício. Em indivíduos treinados, a MLSS não parece ser o limite superior do domínio pesado, pois quando o exercício é realizado acima desta ( 5\%), o $\mathrm{VO}_{2}$ pico não é alcançado ao final de 30 minutos de exercício.

Palavras-chave: Ciclismo; Consumo de oxigênio; Exercício aeróbio.

Abstract - The objective of this study was to determine the validity of maximum lactate steady state (MLSS) for the identification of the upper limit of the heavy-intensity domain in well-trained cyclists. Fourteen male cyclists $(25.5 \pm 4.4$ years, $69.5 \pm 7.8 \mathrm{~kg}, 175.8 \pm 7.5 \mathrm{~cm})$ underwent the following tests on different days: incremental exercise test until exhaustion to determine peak oxygen uptake ( $\mathrm{VO}_{2}$ peak), and 2 to 4 constant submaximal load tests to determine $\mathrm{MLSS} . \mathrm{VO}_{2}$ obtained in the 30th min was significantly higher than that obtained in the 3rd min of exercise performed at $100 \%$ MLSS $(3379.3 \pm 250.1$ vs. $3496.7 \pm 280.2 \mathrm{ml} / \mathrm{min}, p<0.05)$ and $105 \%$ MLSS $(3439.5 \pm 289.3$ vs. $3545.5 \pm 303.2 \mathrm{ml} / \mathrm{min}, p<0.05)$. VO, obtained in the 30 th min of exercise performed at $100 \%$ and $105 \%$ MLSS was significantly lower than $\mathrm{VO}_{2}$ peak $(3978.6 \pm$ $296.2 \mathrm{ml} / \mathrm{min}, \mathrm{p}>0.05$ ). These results suggest that during heavy exercise (MLSS) $\mathrm{VO}_{2}$ does not present stability when values obtained around the $3 r d$ minute of exercise are used as reference. MLSS does not seem to be the upper limit of the heavy-intensity domain in trained subjects since $\mathrm{VO}_{2}$ peak is not reached by the end of $30 \mathrm{~min}$ of exercise when exercise is performed above this intensity $(\sim 5 \%)$.

Key words: Cycling; Oxygen uptake; Aerobic exercise. 


\section{INTRODUÇÃO}

O exercício pode ser realizado em diferentes domínios de intensidade, definidos com base em repostas metabólicas específicas. Os domínios de intensidade são classificados como moderado, pesado e severo e em cada um destes, as respostas ao longo do tempo da concentração de lactato sanguíneo ([La]), $\mathrm{pH}$ e consumo de oxigênio $\left(\mathrm{VO}_{2}\right)$ são diferentes ${ }^{1,2}$. O exercício moderado [i.e., abaixo do limiar de lactato (LL)] é altamente sustentável, sem aumento contínuo na [La]. No domínio pesado, que inclui as intensidades acima do LL, mas abaixo da máxima fase estável de lactato (MLSS) ou potência crítica (PC), a [La] tende a se estabilizar também, porém em uma concentração maior em relação ao domínio moderado. Além disso, há o surgimento do componente lento do $\mathrm{VO}_{2}(\mathrm{CL})$, que faz com que este se estabilize em um valor maior que o predito pela relação sub- $\mathrm{LLVO}_{2}$-carga. Já nas intensidades de exercício correspondentes ao domínio severo (i.e., acima da MLSS ou PC) $)^{3}$, a [La] e o $\mathrm{VO}_{2}$ aumentam progressivamente, sendo que o aumento do $\mathrm{VO}_{2}$ pode ser na forma biexponencial, pelo desenvolvimento do CL em intensidades abaixo do pico do consumo de oxigênio $\left(\mathrm{VO}_{2}\right.$ pico $)$ ou este é projetado exponencialmente (em intensidades no ou acima do $\mathrm{VO}_{2}$ pico), alcançando o valor máximo ao final do exercício ${ }^{4}$. Além dos diferentes comportamentos do CL entre os domínios, sua amplitude é maior no domínio severo do que no domínio pesado ${ }^{2,3}$. Deste modo, o limite superior do domínio pesado (i.e., MLSS ou PC) representa a mais alta taxa de trabalho que pode ser sustentada sem um progressivo aumento na contribuição anaeróbia ${ }^{5}$.

Assim, a acurácia na determinação dos limites dos domínios de intensidade de exercício (LL, MLSS e PC) tem sido considerada essencial tanto para a prescrição do exercício aeróbio, bem como na elaboração de delineamentos experimentais. Entretanto, analisando-se os dados existentes na literatura, bem como os conceitos teóricos que procuram sustentar qual seria o limite entre o domínio pesado e severo, percebe-se a existência de informações que são aparentemente antagônicas.

Poole et al..$^{5}$ e Hill et al. ${ }^{6}$ têm proposto que a PC seria o limite superior do domínio pesado, pois estes autores verificaram que nesta intensidade o $\mathrm{VO}_{2}$ não atingiu seu valor máximo, apresentando ainda, em muitos casos, um valor estável. Entretanto, quando o exercício foi realizado um pouco acima da PC (5 - 10\%), o $\mathrm{VO}_{2}$ pico foi alcançado e sustentado. Por outro lado, baseados em seus achados, Pringle e Jones ${ }^{3}$ sustentam que a MLSS e não a $\mathrm{PC}$, seria o limite entre o domínio pesado e severo. Neste estudo, os autores verificaram que a PC foi significantemente maior que a MLSS, e que se exercitando acima da MLSS ( 20W), a [La] e $\mathrm{O}_{2}$ não apresentaram estabilidade. Além disso, outros autores ao elaborarem seus delineamentos, hipóteses e discussão dos resultados, frequentemente usam como similares as intensidades correspondentes a PC e a MLSS?

Entretanto, deve-se apontar que diversos estudos realizados no ciclismo estacionário ${ }^{3,8}$, corrida $^{9}$ e natação $0^{10}$ têm verificado que a $\mathrm{PC} /$ velocidade crítica é significantemente correlacionada, porém maior que a MLSS. Deste modo, a PC e a MLSS representam diferentes intensidades de exercício, podendo apresentar respostas agudas ao esforço ([La], $\mathrm{VO}_{2}$ e tempo de exaustão) também distintas e potencialmente não podem ser utilizadas de modo intercambiável. Assim, considerando que a PC possa ser o limite superior do domínio pesado ${ }^{5,6}$, pode-se hipotetizar que existem intensidades de exercício (i.e., > MLSS e $\leq \mathrm{PC}$ ) nas quais o lactato sanguíneo não apresentaria estabilidade ao longo do tempo, o CL seria desenvolvido (a intensidade é maior que o LL), mas o $\mathrm{VO}_{2}$ pico não seria alcançado antes do indivíduo entrar em exaustão. Com base nestes pressupostos, pode ser interessante analisar as respostas metabólicas dos indivíduos se exercitando na MLSS e logo acima desta, para que se investigue mais detalhadamente dois importantes aspectos: 1) a validade da MLSS para identificar o limite superior do domínio pesado de exercício e; 2) as relações temporais entre a [La] e o surgimento do CL que leve ou não à obtenção do $\mathrm{VO}_{2}$ pico durante duas condições metabólicas bem distintas (com e sem fase estável de lactato sanguíneo). Este último aspecto tem especial interesse teórico, pois ainda existem controvérsias em relação aos fatores que determinam o $\mathrm{CL}^{1}$, com alguns autores sustentando que a relação entre a resposta de lactato sanguíneo e CL é apenas temporal e não de causa-efeito.

Um modelo interessante de estudo para testar os pressupostos descritos anteriormente, seria analisar as respostas metabólicas ([La] e $\mathrm{VO}_{2}$ ) na MLSS e logo acima desta, em indivíduos com elevada aptidão aeróbia. Nestes indivíduos, em função dos efeitos do treinamento aeróbio e do maior percentual de fibras do Tipo I, o CL é potencialmente menor, exigindo uma intensidade maior de exercício para que o $\mathrm{VO}_{2}$ pico seja atingido durante o exercício de carga constante. Com isso, nós hipotetizamos que em indivíduos com elevada aptidão aeróbia a 
MLSS não delimita o domínio pesado de exercício, ou seja, embora exista aumento da [La] ao longo do tempo, a amplitude do CL não é suficiente para que o $\mathrm{VO}_{2}$ pico seja atingido. Desse modo, o objetivo deste estudo foi verificar se a MLSS representa o limite superior do domínio pesado em ciclistas bem treinados.

\section{PROCEDIMENTOS METODOLÓGICOS}

\section{Sujeitos}

Participaram do estudo quatorze ciclistas do sexo masculino (25,5 \pm 4,4 anos, $69,5 \pm 7,8 \mathrm{~kg}, 175,8 \pm$ $7,5 \mathrm{~cm}$ ), com pelo menos 5 anos de experiência na modalidade. Os atletas treinavam de 6 a 7 vezes por semana (volume $=403 \pm 50 \mathrm{~km}$ ) e participavam de competições em nível estadual e nacional. Os indivíduos foram orientados a não realizarem treinamento intenso e não ingerirem bebidas contendo cafeína e álcool nas 24 horas que antecederam as sessões experimentais. Este estudo foi aprovado pelo Comitê de Ética em Pesquisa da Universidade Estadual Paulista - UNESP (Campus Rio Claro).

\section{Delineamento experimental}

Os atletas compareceram ao laboratório entre 3 e 5 vezes, dentro de um período de 2 semanas. Inicialmente, os atletas realizaram um teste incremental para determinar o limiar anaeróbio (LAn) e o pico do consumo de oxigênio $\left(\mathrm{VO}_{2}\right.$ pico). Durante as demais sessões, os atletas realizaram exercícios de carga constante para determinar a MLSS. Os testes foram separados por intervalos de, pelo menos, $48 \mathrm{~h}$, e foram realizados nos mesmos horários do dia $( \pm 2 \mathrm{~h})$ em um laboratório com temperatura controlada $\left(21-22^{\circ} \mathrm{C}\right)$.

\section{Protocolo incremental}

Os sujeitos foram submetidos a exercício incremental em cicloergômetro de frenagem eletromagnética (Excalibur Sport, Lode BV, Croningen, Holanda) com frequência de pedalada entre 70 e $90 \mathrm{rpm}$. A intensidade inicial foi de $1,5 \mathrm{~W} \cdot \mathrm{kg}^{-1} \mathrm{com}$ incrementos de $0,5 \mathrm{~W} \cdot \mathrm{kg}^{1}$ de massa corporal a cada 3 minutos até a exaustão voluntária. Ao final de cada estágio, houve coleta de sangue do lóbulo da orelha para a dosagem do lactato sanguíneo (YSL 2300 STAT, Yellow Springs, Ohio, EUA). O VO foi mensurado respiração a respiração, durante todo o protocolo, a partir do gás expirado (Quark PFTergo - Cosmed, Itália), sendo os dados reduzidos às médias de $15 \mathrm{~s}$. A calibração dos sistemas de análise das concentrações de $\mathrm{O}_{2}$ e $\mathrm{CO}_{2}$ foi feita antes de cada teste, usando ar ambiente e um gás com concentrações conhecidas de $\mathrm{O}_{2}$ e $\mathrm{CO}_{2}$ de acordo com as instruções do fabricante. A turbina do analisador foi calibrada através de uma seringa de $31 . \mathrm{O} \mathrm{VO}_{2}$ pico foi considerado como o maior valor de $15 \mathrm{~s}$ obtido durante o teste. O LAn foi encontrado através de uma interpolação linear (lactato vs. intensidade), considerando uma concentração fixa de lactato de $3,5 \mathrm{mM}^{11}$.

\section{Protocolo de carga constante}

Foram realizados de dois a quatro testes de carga constante, com duração de 30 minutos, em diferentes intensidades, para a determinação da potência (MLSSp), [La], frequência cardíaca (FC) e $\mathrm{VO}_{2}$ correspondentes a 100 e $105 \%$ MLSS. A primeira carga foi equivalente a $105 \%$ do LAn. No 10 e e 30 o minuto dos testes foram coletadas amostras de sangue do lóbulo da orelha para a determinação da [La]. Para a análise do $\mathrm{VO}_{2}$, foram considerados os valores médios obtidos de 2,75으 ao 3,25 minuto e $29,5 \circ$ ao $30^{\circ}$ minuto dos testes. A MLSS foi considerada como sendo a mais alta carga na qual foi observado um aumento menor ou igual a $1,0 \mathrm{mM}$ entre o 10 e 30 minuto de exercício, conforme proposto por Beneke ${ }^{12}$.

\section{Análise Estatística}

Os dados estão expressos como média \pm DP. A normalidade dos dados foi verificada através do teste de Shapiro-Wilk. Os valores de [La], $\mathrm{VO}_{2}$ e FC obtidos durante o exercício de carga constante, e também entre as diferentes intensidades foram comparados através do teste $t$ de Student para dados pareados. O nível de significância foi mantido em $\mathrm{p} \leq 0,05$.

\section{RESULTADOS}

As variáveis obtidas no teste incremental estão apresentadas na Tabela 1. O LAn correspondeu a $74,9 \%$ da potência máxima atingida no teste incremental $(P \max )$ e a $87,3 \%$ FCmax.

Tabela 1. Valores médios \pm DP das variáveis obtidas no protocolo incremental. $\mathrm{N}=14$

\begin{tabular}{lc}
\hline & Média \pm DP \\
\hline Pmax $(\mathrm{W})$ & $360,5 \pm 33,9$ \\
$\mathrm{VO}_{2}$ pico $\left(\mathrm{ml} \cdot \mathrm{kg}^{-1} \cdot \mathrm{min}^{-1}\right)$ & $58,3 \pm 6,9$ \\
FCmax $(\mathrm{bpm})$ & $189,0 \pm 9,18$ \\
PLAn $(\mathrm{W})$ & $270,0 \pm 34,2$ \\
FCLAn $(\mathrm{bpm})$ & $165,0 \pm 8,4$ \\
\hline
\end{tabular}

Pmax - potência máxima; $\mathrm{VO}_{2}$ pico - pico do consumo de oxigênio; FCmax - frequência cardíaca máxima; PLAn - potência no limiar anaeróbio; FCLAn - frequência cardíaca no limiar anaeróbio. 
A Tabela 2 apresenta os valores médios \pm DP da MLSSp, \%Pmax, [La], VO, FC e \%FCmax obtidos durante o exercício de carga constante realizado a $100 \%$ MLSS e $105 \%$ MLSS. Todas as variáveis obtidas a 105\%MLSS foram estatisticamente maiores $(\mathrm{p}<0,05)$ do que a 100\%MLSS. Houve estabilidade da [La] somente a $100 \%$ MLSS.

Tabela 2. Valores médios \pm DP da potência (MLSSp), concentração de lactato sanguíneo ([La]) e consumo de oxigênio $\left(\mathrm{VO}_{2}\right)$ obtidos no exercício contínuo realizado a 100\%MLSS e $105 \%$ MLSS. $\mathrm{N}=14$

\begin{tabular}{|c|c|c|}
\hline & $100 \%$ MLSS & $105 \%$ MLSS \\
\hline MLSSp (W) & $267,2 \pm 27,4$ & $278,2 \pm 29,5^{*}$ \\
\hline$\%$ Pmax & 74,2 & $78,2^{*}$ \\
\hline$\% F C \max$ & $88,0 \pm 4,1$ & $90,7 \pm 3,5^{*}$ \\
\hline$\% \mathrm{VO}_{2} \max$ & $85,3 \pm 4,2$ & $88,7 \pm 8,4^{*}$ \\
\hline [La] 10ㅇ (mM) & $3,8 \pm 1,6$ & $5,5 \pm 1,4$ \\
\hline [La] 30(mM) & $4,2 \pm 1,6$ & $7,7 \pm 1,9^{+}$ \\
\hline $\mathrm{VO}_{2} 3^{\circ} \min \left(\mathrm{ml}^{\left.-\mathrm{min}^{-1}\right)}\right.$ & $3379,3 \pm 250,1$ & $3439,5 \pm 289,3^{*}$ \\
\hline $\mathrm{VO}_{2} 30^{\circ} \min \left(\mathrm{ml} \cdot \mathrm{min}^{-1}\right)$ & $3496,7 \pm 280,2^{+}$ & $3545,5 \pm 303,2 *($ \\
\hline
\end{tabular}

* P<0,05 em relação a 100\%MLSS. ( $\mathrm{P}<0,05$ em relação ao $10^{\circ}$ $\min ([\mathrm{La}])$ e 30 min $\left(\mathrm{VO}_{2}\right)$ na mesma intensidade.

Os valores do $\mathrm{VO}_{2}$ obtidos no $30^{\circ}$ min de exercício foram estatisticamente maiores $(\mathrm{p}<0,05)$ do que os valores obtidos no 3 o min de exercício a $100 \%$ MLSS e a $105 \%$ MLSS. No entanto, embora os valores obtidos a $105 \%$ MLSS tenham sido maiores que os valores obtidos a $100 \%$ MLSS, o delta de variação dos mesmos foi similar $(3,1 \%$ e $3,4 \%$, respectivamente). $\mathrm{O} \mathrm{VO}_{2}$ obtido no $30 \circ \mathrm{min}$ no exercício realizado tanto a $100 \%$ MLSS quanto a 105\%MLSS foi significantemente menor do que o $\mathrm{VO}_{2}$ pico $(\mathrm{p}<0,05)$. A Figura 1 apresenta o comportamento da [La] e do $\mathrm{VO}_{2}$ durante o exercício realizado a $100 \%$ MLSS e a $105 \%$ MLSS de um ciclista representativo.

\section{DISCUSSÃO}

O principal achado deste estudo foi que o comportamento do $\mathrm{VO}_{2}$ não parece indicar que a MLSS seja o limite superior do domínio pesado, já que, mesmo exercitando-se acima desta intensidade, o $\mathrm{VO}_{2}$ pico não foi atingido ao final de 30 minutos de exercício.

Analisando-se os valores correspondentes à MLSS obtidos neste estudo, as intensidades absoluta e relativa são próximas às obtidas por Denadai et al. ${ }^{11}$ (282 W, 79\% Pmax), em ciclistas treinados. Em um outro estudo, Baron et al. ${ }^{13}$ verificaram que a MLSS correspondeu a $71 \%$ do $\mathrm{VO}_{2}$ pico
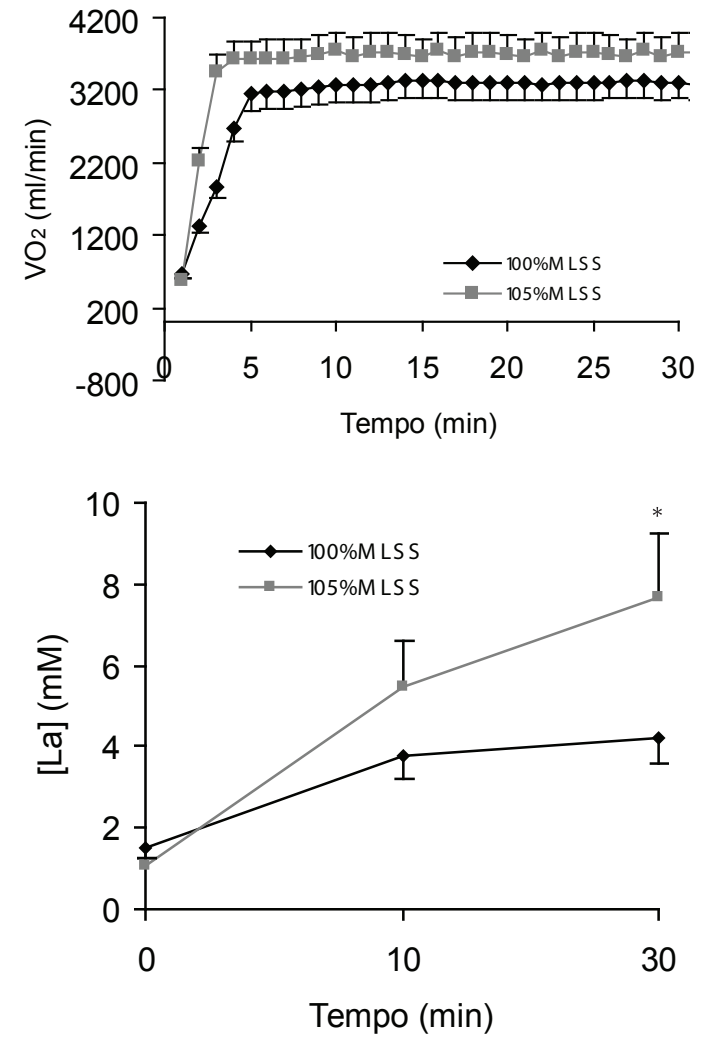

Figura 1. Valores médios \pm DP do consumo de oxigênio $\left(\mathrm{VO}_{2}\right)$ e da concentração de lactato sanguíneo ([La]) durante o exercício realizado a 100\%MLSS e a 105\%MLSS. * p < 0,05 em relação à $100 \%$ MLSS.

em indivíduos treinados. No estudo realizado por Denadai et al. ${ }^{11}$, os valores obtidos no grupo de indivíduos sedentários foram de $180 \mathrm{~W}$ e $68 \%$ Pmax, respectivamente. Estes dados, juntamente com dados obtidos por estudos que verificaram altas intensidades absolutas e relativas correspondentes ao $\mathrm{LL}$ em indivíduos treinados ${ }^{14}$, sugerem que o treinamento aeróbio desloca os índices limítrofes dos domínios em direção ao $\mathrm{VO}_{2}$ pico, aumentando o domínio moderado e reduzindo o domínio pesado de exercício. Como a MLSS representa uma intensidade na qual a taxa de produção de energia aeróbia é bastante elevada e esta ocorre com grande participação da glicólise, as elevadas intensidades relativas encontradas em indivíduos treinados sugerem que estes têm uma maior habilidade de sustentar altos níveis de esforço ainda mantendo estabilidade na [La].

O comportamento da [La] encontrado neste estudo concorda com os dados obtidos por diversos estudos $^{3,8,10,12}$ que verificaram estabilidade desta variável a $100 \%$ MLSS, porém um aumento contínuo ao exercitar-se acima dela. Este aumento contínuo representa um aumento da participação do metabolismo anaeróbio e uma incapacidade de manter a 
taxa de remoção do lactato sanguíneo similar à taxa de formação do mesmo. Com base nestes dados, sugere-se que além da MLSS representar a mais alta intensidade em que haveria equilíbrio entre a produção e remoção do lactato sanguíneo, ela poderia ser também considerada o índice limítrofe entre os domínios pesado e severo de exercício 3 .

Como esperado, as repostas do $\mathrm{VO}_{2}$ durante o exercício realizado a 105\%MLSS foram significantemente maiores do que a 100\%MLSS. Porém, houve um aumento similar nas duas intensidades entre o 3 e o 30 min de exercício (3,1\% e 3,4\%, respectivamente). Nós optamos por analisar a variação do $\mathrm{VO}_{2}$ a partir do 3ำ minuto, em função de muitos estudos mostrarem que o CL emerge a partir de, aproximadamente, o 2 minuto de exercício ${ }^{15}$. A amplitude do CL encontrado em nosso estudo (3,1\% e 3,4\%, respectivamente) é aparentemente menor que a encontrada por Carter et al..$^{15}$ durante o exercício realizado por indivíduos ativos no domínio pesado (6 a 15\%). Os menores valores observados em nosso estudo concordam com dados encontrados na literatura que verificaram menores CL em indivíduos treinados ${ }^{16,17}$.

Diferentemente do nosso estudo, Pringle e Jones ${ }^{3}$ e Baron et al. ${ }^{13}$ verificaram estabilidade do $\mathrm{VO}_{2}$ durante exercícios realizados na MLSS. Entretanto, nestes estudos $\mathrm{O}_{2}$ foi analisado apenas a partir do $10 \circ$ minuto de exercício. Alguns autores apontam que, no exercício realizado no domínio pesado, o CL desenvolve-se mais rapidamente nos primeiros minutos do exercício ( $3 \mathrm{~min}$ a $10 \mathrm{~min})^{1}$ apresentando posteriormente estabilidade do $\mathrm{VO}_{2}$. Assim, é provável que estes diferentes resultados possam ser explicados pelos diferentes intervalos de análise do $\mathrm{VO}_{2}$ utilizado em nosso estudo (3으 $30^{\circ} \mathrm{min}$ ) e por Pringle e Jones ${ }^{3}$ e Baron et al..$^{13}$ (> 10 x 30 min).

Quando se analisa o $\mathrm{VO}_{2}$ atingido ao final do exercício realizado a 105\%MLSS, verificou-se que o mesmo foi significantemente menor ( $89 \%$ $\mathrm{VO}_{2}$ pico) do que o $\mathrm{VO}_{2}$ pico. Este dado concorda com o obtido por Pringle e Jones ${ }^{3}$, que verificaram um valor de $89 \%$ do $\mathrm{VO}_{2}$ pico quando os indivíduos exercitaram-se acima da MLSS. Desta forma, sugere-se que a MLSS não seja o índice fisiológico que delimita o domínio pesado do severo, pois embora não tenha existido estabilidade da [La], os indivíduos não atingiram o $\mathrm{VO}_{2}$ pico ao final de 30 minutos de exercício. Além disso, os valores similares de variação do $\mathrm{VO}_{2}$ entre $100 \%$ MLSS e 105\%MLSS encontrados neste estudo, podem também sugerir que os indivíduos exercitaram-se no mesmo domínio de exercício. Interessantemente, a ausência de estabilidade do lactato sanguíneo não parece estar associada nem mesmo temporalmente com a existência do CL que determine que o $\mathrm{VO}_{2}$ pico seja atingido durante o exercício de carga constante. Na verdade, intensidades de exercício com (100\%MLSS) e sem (105\%MLSS) estabilidade de lactato, nas condições experimentais deste estudo, produziram amplitudes de CL similares.

Uma possível limitação deste estudo é o fato de não termos levado os ciclistas até a exaustão voluntária, particularmente, durante o exercício realizado a 105\%MLSS, analisando-se nestas condições os valores de $\mathrm{VO}_{2}$. Entretanto, alguns aspectos devem ser apontados, para que se verifique o nível desta limitação. Primeiro, Baron et al. ${ }^{13}$ verificaram em indivíduos treinados um tempo de exaustão médio de 55 min durante o exercício a 100\%MLSS. Baseado na relação hiperbólica entre a intensidade de esforço e tempo de exaustão ${ }^{4}$, é possível esperar que o tempo máximo de exercício a 105\%MLSS, não seja muito superior aos 30 minutos analisados no presente estudo. Confirmando este aspecto, Pringle e Jones ${ }^{3}$ verificaram que $50 \%$ dos seus voluntários (indivíduos ativos e treinados) não completaram 30 min de esforço, durante o exercício realizado logo acima $(\sim 8 \%)$ da MLSS. Além disto, a amplitude do CL encontrado em nosso estudo, no exercício acima da MLSS (3,4\%), é menor do que aquele observado em intensidades de carga constante que levam ao $\mathrm{VO}_{2}$ pico $(>10 \%)^{15,18}$.

\section{CONCLUSÃO}

Com base nestes resultados, pode-se concluir que durante o exercício pesado (MLSS) o $\mathrm{VO}_{2}$ não apresenta estabilidade, tomando como referência os valores obtidos por volta do 3o minuto de exercício. Em indivíduos treinados a MLSS não parece ser o limite superior do domínio pesado, pois quando o exercício é realizado acima desta $(\sim 5 \%)$, o $\mathrm{VO}_{2}$ pico não é alcançado ao final de 30 minutos de exercício. Assim, não parece ser possível associar-se o conceito de estabilidade ou não da [La] e o desenvolvimento do CL que determine o alcance ou não do $\mathrm{VO}_{2}$ pico durante o exercício de carga constante.

\section{REFERÊNCIAS BIBLIOGRÁFICAS}

1. Gaesser GA, Poole DC. The slow component of oxygen uptake kinetics in humans. Exerc Sport Sci Rev 1996;24(1):35-70.

2. Carter H, Pringle JS, Jones AM, Doust JH. Oxygen uptake kinetics during treadmill running across exercise intensity domains. Eur J Appl Physiol 2002;86(1-2):347-354 . 
3. Pringle JS, Jones AM. Maximal lactate steady state, critical power and EMG during cycling. Eur J Appl Physiol 2002;88(3):214-226.

4. Caputo F, Denadai BS. The highest intensity and the shortest duration permitting attainment of maximal oxygen uptake during cycling: effects of different methods and aerobic fitness level. Eur J Appl Physiol 2008;103(1):47-47.

5. Poole DC, Ward SA, Gardner G, Whipp BJ. Metabolic and respiratory profile of the upper limit for prolonged exercise in man. Ergonomics 1988;31(9):1265-1279.

6. Hill DW, Poole DC, Smith JC. The relationship between power and time to achieve VO2max. Med Sci Sports Exerc 2002;34(4):709-714.

7. Burnley M, Doust JH, Vanhatalo A. A 3-min all-out test to determine peak oxygen uptake and the maximal steady state. Med Sci Sports Exerc 2006;38(11):1995-2003.

8. Dekerle J,. Baron B, Dupont L, Vanvelcenaher J, Pelayo P. Maximal lactate steady state, respiratory compensation threshold and critical power Eur J Appl Physiol 2003;89(3-4): 281-288.

9. Denadai BS, Gomide EBG, Greco CC. The relationship between onset of blood lactate accumulation, critical velocity and maximal lactate steady state in soccer players. J Strength Cond Res 2005;19(2):364-368.

10. Dekerle J, Pelayo P, Clipet B, Depretz S, Lefevre T, Sidney M. Critical swimming speed does not represent the speed at maximal lactate steady state. Int J Sports Med 2005;26(7):524-530.

11. Denadai BS, Figuera, TR, Favaro ORP, Gonçalves M. Effect of the aerobic capacity on the validity of the anaerobic threshold for determination of the maximal lactate steady state in cycling. Braz J Med Biol Res 2004;37(10):1551-1556.
12. Beneke R. Maximal lactate steady state concentration (MLSS): experimental and modelling approaches. Eur J Appl Physiol 2003;8(4-5):361-369.

13. Baron B, Noakes TD, Dekerle J, Moullan F, Robin S, Matran R, et al. Why does exercise terminate at the maximal lactate steady state intensity? Br J Sports Med 2008;42(10):528-533

14. Joyner MJ, Coyle EF. Endurance exercise performance: the physiology of champions. J Physiol 2008;586(1):35-44.

15. Carter H, Jones AM, Barstow TJ, Burnley M, Williams $\mathrm{CA}$, Doust JH. Oxygen uptake kinetics in treadmill running and cycle ergometry: a comparison. J Appl Physiol 2000;89(3):899-907.

16. Casaburi R, Storer TW, Ben-Dov I, Wasserman K. Effect of endurance training on possible determinants of VO2 during heavy exercise. J Appl Physiol 1987;62(1):199-207.

17. Poole DC, Ward SA, Whipp BJ. The effects of training on the metabolic and respiratory profile of highintensity cycle ergometer exercise. Eur J Appl Physiol 1990;59(6):421-429.

18. Hill DW, Halcomb JN, Stevens EC. Oxygen uptake kinetics during severe intensity running and cycling. Eur J Appl Physiol 2003;89(6):612-618.

\section{Endereço para correspondência}

Camila Coelho Greco

Instituto de Biociências, Laboratório de Avaliação da Performance Humana

Bela Vista, Avenida 24A, 1515

13506-900 - Rio Claro, SP. Brasil

E-mail: grecocc@rc.unesp.br 